\title{
Mortalidade infantil em Mato Grosso, Brasil: tendência entre 2007 e 2016 e causas de morte
}

\author{
Infant mortality in Mato Grosso, Brazil: trend and causes of death \\ between 2007 and 2016
}

Angélica Fátima Bonatti (https://orcid.org/0000-0003-3327-393X) ${ }^{1}$

Ageo Mario Cândido da Silva (https://orcid.org/0000-0001-5293-9413) ${ }^{1}$

Ana Paula Muraro (https://orcid.org/0000-0001-6237-1673) ${ }^{1}$

${ }^{1}$ Universidade Federal de Mato Grosso. R. Quarenta e Nove 2367, Boa Esperança. 78060-900 Cuiabá MT Brasil.angelica.bonatti.ab@ gmail.com

\begin{abstract}
Geographical and temporal variations in infant mortality subsidize the process of planning and assessing maternal and child health. This is a time-series study that set out to analyze the trend of infant mortality in Mato Grosso and its health regions from 2007 to 2016 and to identify the distribution of causes of death according to age group and birth weight. For the trend analysis, the infant mortality rates stratified by causes of death between 2007 and 2016 were evaluated using polynomial regression models. There was a decreasing linear trend in infant mortality rates in the state, but individually only four of the 16 health regions followed this trend. Half of the deaths occurred in the early neonatal period. Causes avoidable by adequate attention to women during pregnancy and childbirth and to the newborn represented $51.1 \%$ of the deaths. There was an increasing tendency of proportional mortality for causes not manifestly avoidable. It is acknowledged that it is important to know the trend of rates in order to define priority population groups for early interventions that increase survival and reduce infant mortality. In addition, the investigation of deaths in the state should be implemented to establish the underlying cause.
\end{abstract}

Key words Infant mortality, Cause of death, Regression analysis
Resumo As variações geográficas e temporais da mortalidade infantil subsidiam o processo de planejamento e avaliação da saúde materno-infantil. Estudo de série temporal que objetivou analisar a tendência da mortalidade infantil em Mato Grosso e regiões de saúde, no período de 2007 a 2016 e identificar a distribuição das causas de morte, segundo grupo etário e peso ao nascer. Para a análise de tendência, avaliou-se as taxas de mortalidade infantil estratificadas por causas de morte, entre 2007 e 2016, por meio de modelos de regressão polinomial. Foi observada tendência linear decrescente das taxas de mortalidade infantil no estado, porém individualmente, apenas quatro das 16 regiões de saúde seguiram essa tendência. Metade dos óbitos ocorreu no período neonatal precoce. As causas reduzíveis por adequada atenção à mulher na gestação e parto e ao recémnascido representaram $51,1 \%$ dos óbitos. Houve tendência crescente da mortalidade proporcional por causas não claramente evitáveis. Entende-se, que é importante conhecer a tendência das taxas, de forma a definir populações prioritárias para intervenções precoces que aumentem a sobrevida $e$ reduzam a mortalidade infantil. Além disso, devese implementar a investigação de óbito no estado para qualificar a causa básica.

Palavras-chave Mortalidade infantil, Causa de morte, Análise de regressão 


\section{Introdução}

O diagnóstico da situação de saúde materno infantil é considerado o principal instrumento para identificação da magnitude dos problemas a serem enfrentados. Nesse sentido, os indicadores de saúde contribuem para esse processo, pois são utilizados no acompanhamento das flutuações e tendências históricas do padrão sanitário de diferentes coletividades. Um dos indicadores mais utilizados na mensuração da situação de saúde de uma população é a mortalidade infantil ${ }^{1}$.

A análise do óbito infantil segundo grupos de causa permite a avaliação da qualidade das políticas públicas relacionadas à saúde da criança, mas também do cuidado oferecido à gestante durante o pré-natal e no momento do parto². Além disso, orienta na identificação de grupos populacionais mais expostos ao risco de morrer por causas evitáveis, dessa forma, coeficientes elevados refletem, em geral, níveis precários de saúde, condições de vida e desenvolvimento socioeconômico ${ }^{3}$.

Os óbitos evitáveis são considerados eventos sentinela, o que significa dizer que a sua ocorrência é resultado de falhas no processo de prevenção, diagnóstico e tratamento do evento ou da condição que o determina ${ }^{4-6}$. Devido ao potencial de evitabilidade dos óbitos infantis, a Organização Mundial de Saúde (OMS) ${ }^{7}$, considera que cerca de 75\% dos óbitos em recém-nascidos poderiam ser evitados se fossem tomadas medidas de saúde eficazes durante a gestação, no momento do nascimento e na primeira semana de vida.

No Brasil, houve a redução de $70 \%$ na TMI entre $1990(47,1 / 1.000 \mathrm{NV})$ e $2015(12,4 / 1.000$ $\mathrm{NV}$ ), porém, com distribuição desigual entre as regiões do país, observando-se redução mais acentuada na Região Nordeste (78,5\%), seguida da Região Sudeste (62,2\%), e mais discreta na Região Centro-Oeste $(56,1 \%)^{8}$. O país atingiu a meta dos ODM para redução de $2 / 3$ da mortalidade infantil antes do prazo final de $2015^{9}$, contudo, após um longo período de redução da mortalidade infantil, foi verificado o aumento de $2,4 \%$ neste coeficiente, sendo estimado em 12,7/1.000 $\mathrm{NV}$ em $2016^{10}$. Esse aumento é explicado em parte pela diminuição no número de nascidos vivos nesse período e pelo aumento de óbitos no período pós-neonatal em consequência da exposição a fatores externos associados à piora das condições de vida e do acesso a serviços de saúde ${ }^{10}$.

O estado de Mato Grosso, situado na Região-Centro Oeste, reduziu em $50,7 \%$ a taxa de mortalidade infantil entre 1990 (37,5/1.000 NV) a $2015(18,5 \%)^{8}$. Ao analisar o processo de regio- nalização em Mato Grosso, os autores ${ }^{11}$ sugerem a existência de desigualdades na distribuição do coeficiente de mortalidade infantil nas Regiões de Saúde devido à diversidade das características socioeconômicas e de capacidade de saúde instalada.

Dessa forma, torna-se essencial avaliar a situação de saúde da mortalidade infantil de forma regionalizada a fim de identificar sua evolução e definir políticas prioritárias diante as dificuldades enfrentadas pelas regiões, que se diluem, na maioria das vezes, em avaliações mais gerais. Nesse contexto, o objetivo desse estudo foi analisar a tendência da mortalidade infantil em Mato Grosso e regiões de saúde, no período de 2007 a 2016 e identificar a distribuição das causas de morte, segundo grupo etário e peso ao nascer.

\section{Métodos}

Estudo de série temporal com dados do Sistema de Informação sobre Nascido Vivo (Sinasc) e Mortalidade (SIM) em Mato Grosso. O estado é composto por 141 municípios, organizados em 16 regiões de saúde, com população estimada de 3.305.531 habitantes ${ }^{12}$ e Índice de Desenvolvimento Humano (IDH) de 0,725, ocupando o $11^{\circ}$ lugar entre os estados brasileiros ${ }^{13}$. Além disso, possui boa cobertura do SIM e Sinasc sendo superior a $90 \%{ }^{14}$. Demograficamente, Mato Grosso apresenta-se em transição, com redução da natalidade nos últimos vinte anos, diminuição da mortalidade infantil, idade média de 30 anos e discreto envelhecimento populacional ${ }^{11}$.

A Taxa de Mortalidade Infantil (TMI), expressa pelo número de óbitos por mil nascidos vivos, foi calculada pelo método direto, ano a ano, para os grupos de causas e para as regiões de saúde. A causa básica de morte foi extraída das declarações de óbito digitalizadas, fornecidas pela Secretaria Estadual de Saúde (SES-MT). O registro das causas baseou-se na Classificação Internacional de Doenças, 10a revisão (CID-10).

Para a análise da evitabilidade dos óbitos infantis, foi utilizada a "Lista de causas de mortes evitáveis por intervenções no âmbito do Sistema de Saúde do Brasil para menores de cinco anos de idade", proposta por Malta et al. ${ }^{15}$, atualizada em 2010.

Neste estudo, as causas de morte foram organizadas em 4 grupos sendo: Grupo 1 - Causas reduzíveis por adequada atenção a mulher na gestação e parto e ao recém-nascido; Grupo 2 - Causas reduzíveis por ações de diagnóstico, 
tratamento, promoção à saúde e imunoprevenção; Grupo 3 - Causas mal definidas; Grupo 4 - Demais causas, não claramente evitáveis.

A mortalidade proporcional foi calculada segundo os quatro grupos de causas no período de 2007 a 2016 e nas dezesseis regiões de saúde do estado. A proporção dos óbitos segundo grupo de causas também foi analisado por grupo etário, classificado em neonatal precoce ( 0 a 6 dias), neonatal tardio ( 7 a 27 dias) e pós-neonatal (28 a 364 dias); e peso ao nascer de 500 a 1.499 gramas, 1.500 a 2.499 gramas e $\geq 2.500$ gramas e mais.

Para estimar a tendência temporal da evitabilidade dos óbitos infantis no período de 2007 a 2016 foram utilizados modelos de regressão polinomial. Foram testados modelos linear, quadrático e cúbico, sendo considerado capaz de descrever a tendência quando o valor de $\mathrm{p}$ foi $<0,05$. Para encontrar a curva que melhor descrevesse a relação entre as variáveis dependente e independente, foram testados os seguintes modelos de regressão polinomial ${ }^{16}$ : Linear: $y=\beta_{0}+\beta_{1} x$; Quadrática: $y=\beta_{0}+\beta_{1} x+\beta_{2} x^{2}$; Cúbica: $y=\beta_{0}+$ $\beta_{1} x+\beta_{2} x^{2}+\beta_{3} x^{3}$.

Os valores de y e $\mathrm{x}$ representam, respectivamente, as variáveis dependente e independente; $\beta_{0}, \beta_{1}, \beta_{2}$ e $\beta_{3}$ são os coeficientes de regressão. Após a análise, considerou-se, como melhor modelo, aquele que apresentou maior coeficiente de determinação $\left(\mathrm{R}^{2}\right)$. Caso houvesse dois modelos semelhantes, do ponto de vista estatístico, para a mesma localidade, optou-se pelo modelo mais simples, ou seja, de menor ordem ${ }^{16}$.

Todas as informações foram tratadas estatisticamente através do software Statistical Package for the Social Science (SPSS), versão 17. O estudo foi aprovado pelo Conselho de Ética em Pesquisa, do Centro de Ciências da Saúde, Universidade Federal de Mato Grosso.

\section{Resultados}

No período de 2007 a 2016, foram notificados 7.732 óbitos menores de um ano de idade, no estado. Dentre esses óbitos, $9(0,12 \%)$ não tinham a informação de causa básica e em $6(0,08 \%)$ não foi possível avaliar a Região de Saúde devido a ausência do registro do município de residência. Verificou-se que $65,1 \%$ dos óbitos foram considerados evitáveis, sendo que $51,1 \%$ poderiam ter sido evitados com adequada atenção à mulher na gestação e parto e ao recém-nascido (Figura 1).

A taxa média da mortalidade infantil em Mato Grosso foi de 15 por 1.000 nascidos vivos
(NV) entre 2007 e 2016, com tendência decrescente no período $(\mathrm{p}=0,001)$. O maior coeficiente por causas de mortes "reduzíveis por adequada atenção a mulher na gestação e parto e ao recém-nascido" ocorreu em 2008 (8,80/1.000 NV), apresentando tendência decrescente no período avaliado com TMI de 6,96/1.000 NV em 2016 (Tabela 1).

Os óbitos "reduzíveis por adequado diagnóstico, tratamento, ações de promoção e imunização" variou de 2,53 em 2007 para 1,74 em 2016, com tendência decrescente ( $\mathrm{p}$-valor $=0,025)$. Os óbitos por causas mal definidas mantiveram proporções e taxas relativamente baixas. Quando avaliado a mortalidade proporcional por grupo de causas, apenas o grupo das demais causas, não claramente evitáveis, mostrou tendência crescente entre os anos de 2007 e 2016 (Tabela 1).

Ao analisar a tendência da mortalidade infantil segundo regiões de saúde do estado de Mato Grosso, 25\% (quatro regiões) apontaram tendência decrescente para o coeficiente em análise, sendo elas: Baixada Cuiabana, Araguaia Xingu, Garças Araguaia e Noroeste Mato-grossense. A região Noroeste Mato-grossense apresentou maior tendência de redução dos óbitos $(\beta$ : $-0,735$; $\mathrm{R}^{2}: 0,620 ; \mathrm{p}=0,007$ ) (Tabela 2).

Dentre as principais causas de morte evitáveis destacaram-se as causas decorrentes de infecções específicas do período perinatal, os transtornos respiratórios e cardiovasculares específicos do período perinatal e feto e recém-nascido afetados por complicações maternas da gravidez; além da influenza, pneumonia e de doenças bacterianas e intestinais. Em relação às causas mal definidas, prevaleceram as causas desconhecidas de morte e outros problemas originados no período perinatal. O grupo das demais causas, não claramente evitáveis, correspondeu às malformações congênitas (Quadro 1).

Em relação às mortes neonatais precoces, $\mathrm{o}$ grupo de causas "reduzíveis por adequada atenção a mulher na gestação e parto e ao recém-nascido" se destacou por ter ocorrido em 76,3\% dos óbitos infantis. Contudo, no componente pós-neonatal foram observadas as maiores proporções de óbitos por causas evitáveis devido ao adequado diagnóstico, tratamento, ações de promoção e imunização (39,9\%), por causas mal definidas $(5,7 \%)$ e demais causas $(47,7 \%)$ (Tabela 3$)$.

A maior mortalidade proporcional em menores de um ano por grupos de causas estratificados segundo a variável peso ao nascer, ocorreu entre bebês nascidos com peso inferior a 1.499 gramas com destaque para as causas reduzíveis por ade- 


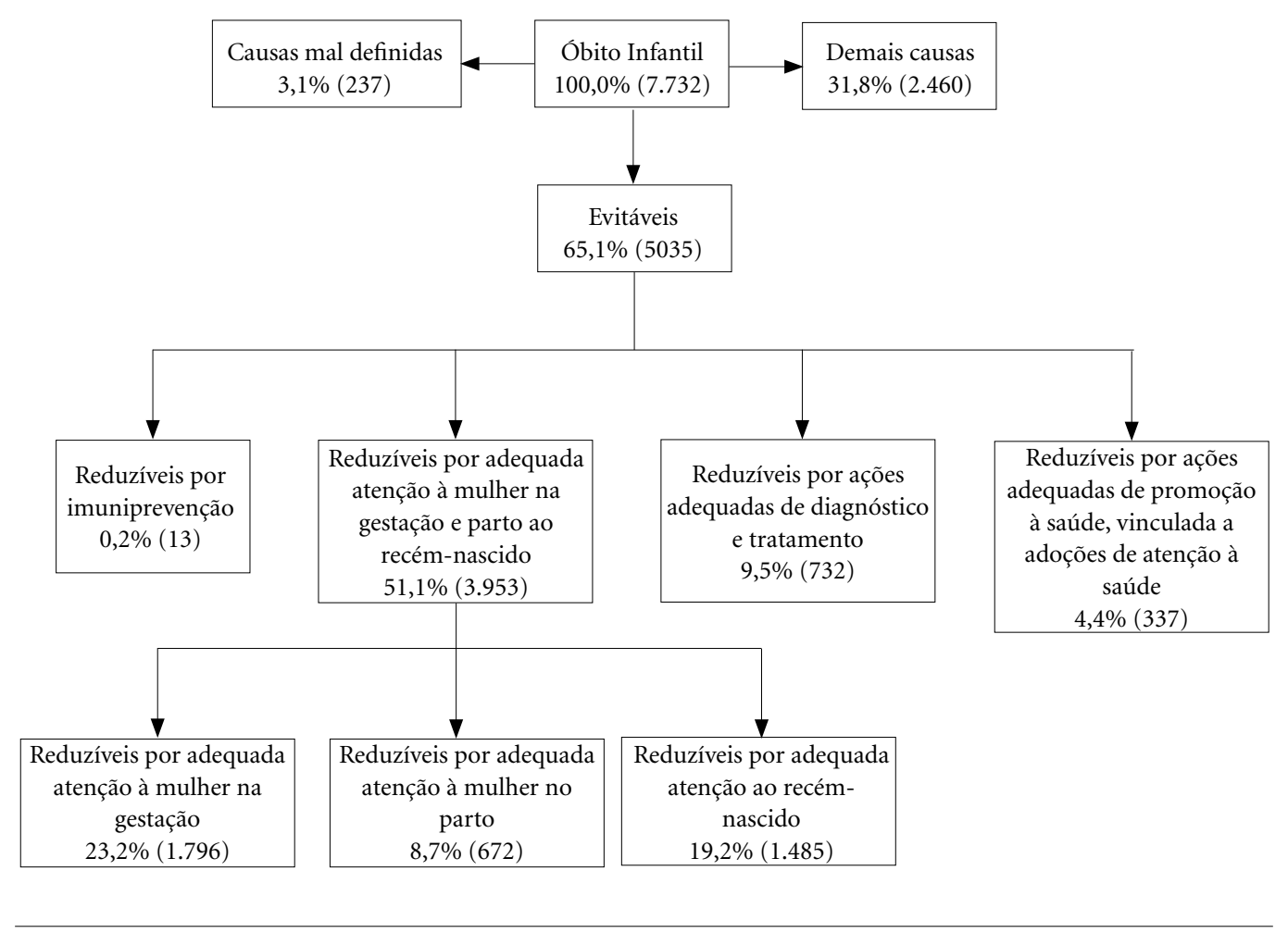

Figura 1. Classificação dos óbitos infantis ${ }^{a}$ pela Lista Brasileira de Causas Evitáveis de Morte. Mato Grosso, Brasil, 2007-2016.

Nota: a Informação faltante para causa básica do óbito para 9 registros $(0,12 \%)$.

quada atenção à mulher na gestação, parto e ao recém-nascido $(79,8 \%)$. Entretanto, verificou-se maior proporção de óbitos pelo grupo de demais causas entre os nascidos com peso adequado (2.500g e mais) (Tabela 3).

\section{Discussão}

Embora tenha ocorrido expressiva redução da mortalidade infantil em Mato Grosso ao longo dos 10 anos, foram encontradas disparidades na tendência da mortalidade infantil entre as regiões de saúde do estado. Além disso, os indicadores mostram elevado risco de mortes precoces decorrente de causas evitáveis, principalmente por adequada atenção a mulher na gestação e parto e ao recém-nascido. Destacou-se também, a tendência crescente da mortalidade proporcional por demais causas, não claramente evitáveis no período analisado.
A tendência decrescente da taxa de mortalidade infantil no estado corrobora a vários estu$\operatorname{dos}^{17-20}$. Diversas estratégias contribuíram para a redução da mortalidade infantil no Brasil e nos estados da federação ao longo dos anos, dentre elas destacam-se ações de promoção à saúde desenvolvida na atenção primária, as políticas de assistência social como o programa "Bolsa Família”, além da melhoria nas condições de vida e na atenção à saúde materna e do recém-nascido ${ }^{21}$.

Esses progressos não beneficiam a população de maneira uniforme Em termos geográficos, por exemplo, as regiões Norte e Nordeste são as mais vulneráveis do Brasil quanto à mortalidade infantil ${ }^{8}$. Em Mato Grosso, também foi verificado desigualdades quanto à distribuição da mortalidade infantil, especialmente na região do Araguaia com expressivo contingente de população indígena ${ }^{22}$.

A etnia indígena dos Xavantes, por exemplo, concentra as maiores taxas de mortalidade infan- 
Tabela 1. Tendência da Taxa de mortalidade infantil (TMI) e da mortalidade proporcional segundo grupo de causas ${ }^{\mathrm{a}}$. Mato Grosso, 2007 a 2016.

\begin{tabular}{|c|c|c|c|c|c|c|c|c|c|}
\hline & \multirow[t]{2}{*}{ TMI } & \multicolumn{2}{|c|}{$\begin{array}{c}\text { Reduzíveis por } \\
\text { adequada atenção à } \\
\text { mulher na gestação } \\
\text { e parto e ao recém- } \\
\text { nascido }\end{array}$} & \multicolumn{2}{|c|}{$\begin{array}{c}\text { Reduzíveis } \\
\text { por adequado } \\
\text { diagnóstico, } \\
\text { tratamento, ações } \\
\text { de promoção e } \\
\text { imunização } \\
\end{array}$} & \multicolumn{2}{|c|}{$\begin{array}{c}\text { Causas mal } \\
\text { definidas }\end{array}$} & \multicolumn{2}{|c|}{ Demais causas } \\
\hline & & $\%$ & TMI & $\%$ & TMI & $\%$ & TMI & $\%$ & TMI \\
\hline 2007 & 16,44 & 52,9 & 8,69 & 15,4 & 2,53 & 3,1 & 0,51 & 28,6 & 4,71 \\
\hline 2008 & 16,3 & 54 & 8,8 & 13,5 & 2,2 & 2,9 & 0,47 & 29,7 & 4,83 \\
\hline 2009 & 16,61 & 49,6 & 8,25 & 18 & 2,99 & 3,6 & 0,59 & 28,8 & 4,78 \\
\hline 2010 & 15,35 & 49,4 & 7,58 & 16,7 & 2,56 & 2,9 & 0,45 & 31 & 4,76 \\
\hline 2011 & 14,41 & 50,9 & 7,34 & 13,2 & 1,91 & 3,5 & 0,51 & 32,3 & 4,65 \\
\hline 2012 & 13,93 & 54 & 7,52 & 10,9 & 1,52 & 4,3 & 0,6 & 30,8 & 4,29 \\
\hline 2013 & 14,56 & 50,1 & 7,29 & 12,4 & 1,81 & 2,6 & 0,38 & 34,9 & 5,09 \\
\hline 2014 & 14,67 & 48 & 7,04 & 14,1 & 2,07 & 2,6 & 0,39 & 35,3 & 5,17 \\
\hline 2015 & 13,81 & 52,2 & 7,21 & 12,6 & 1,75 & 2,3 & 0,32 & 32,8 & 4,53 \\
\hline 2016 & 13,80 & 50,4 & 6,96 & 12,6 & 1,74 & 3 & 0,41 & 34 & 4,69 \\
\hline Tendência $^{\mathrm{b}}$ & Decrescente & - & Decrescente & - & Decrescente & - & - & Crescente & - \\
\hline$\beta^{c}$ & $-0,326$ & $-0,23$ & $-0,203$ & $-0,401$ & $-0,107$ & $-0,062$ & $-0,018$ & 0,685 & 0,002 \\
\hline $\mathrm{R}^{2 \mathrm{~d}}$ & 0,786 & 0,116 & 0,838 & 0,315 & 0,488 & 0,102 & 0,36 & 0,727 & 0,001 \\
\hline p-valor & 0,001 & 0,336 & $<0,001$ & 0,092 & 0,025 & 0,367 & 0,067 & 0,002 & 0,94 \\
\hline
\end{tabular}

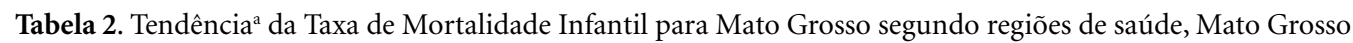
2007-2016.

\begin{tabular}{lrrcc}
\hline \multicolumn{1}{c}{ Região de Saúde } & $\boldsymbol{\beta}^{\mathbf{b}}$ & $\mathbf{R}^{\mathbf{2 c}}$ & p-valor & Tendência \\
\hline Alto Tapajós & $-0,057$ & 0,006 & 0,832 & - \\
Baixada Cuiabana & $-0,279$ & 0,482 & 0,026 & Decrescente \\
Araguaia Xingu & $-1,4$ & 0,568 & 0,012 & Decrescente \\
Centro Norte & 0,087 & 0,004 & 0,866 & - \\
Garças Araguaia & $-1,854$ & 0,591 & 0,009 & Decrescente \\
Médio Araguaia & 0,34 & 0,093 & 0,392 & - \\
Médio Norte Mato-grossense & 0,275 & 0,141 & 0,285 & - \\
Noroeste Mato-grossense & $-0,735$ & 0,62 & 0,007 & Decrescente \\
Norte Araguaia Karajá & 0,111 & 0,001 & 0,931 & - \\
Norte Mato-grossense & $-0,685$ & 0,352 & 0,071 & - \\
Oeste Mato-grossense & $-0,416$ & 0,183 & 0,217 & - \\
Sudoeste Mato-grossense & $-0,668$ & 0,184 & 0,217 & - \\
Sul Mato-grossense & $-0,373$ & 0,302 & 0,1 & - \\
Teles Pires & $-0,176$ & 0,051 & 0,531 & - \\
Vale do Peixoto & 0,608 & 0,293 & 0,106 & - \\
Vale dos Arinos & $-0,372$ & 0,093 & 0,393 & - \\
\hline
\end{tabular}

${ }^{a}$ Modelos de regressão polinomial linear; ${ }^{b} \beta$ : coeficiente de regressão; ${ }^{c} R^{2}$ : coeficiente de determinação.

til do estado, e apresentam como características aldeias de difícil acesso aos serviços de atenção básica em saúde ${ }^{23}$ e, por conseguinte, nascimen- tos e óbitos de crianças sem preenchimento da declaração de óbitos, o que pode ter contribuído para a subnotificação e queda dos coeficientes 
Quadro 1. Mortalidade proporcional, segundo as três principais causas em cada grupo de óbitos infantis, Mato Grosso, 2007 a 2016.

\begin{tabular}{|c|c|c|}
\hline \multirow{2}{*}{ Grupo de causas e CID-10 } & \multicolumn{2}{|c|}{ 2007-2016 } \\
\hline & $\mathbf{n}$ & $(\%)$ \\
\hline \multicolumn{3}{|l|}{ Reduzíveis por adequada atenção à mulher na gestação e parto e ao recém-nascido (P00-P96) } \\
\hline \multicolumn{3}{|l|}{ P35-P39 Infecções específicas do período perinatal } \\
\hline P36.9 Septicemia bacteriana não especificada do recém-nascido & 565 & 14,3 \\
\hline \multicolumn{3}{|l|}{ P20-P29 Transtornos respiratórios e cardiovasculares específicos do período perinatal } \\
\hline P22.0 Síndrome da angústia respiratória do recém-nascido (doença da membrana hialina) & 511 & 12,9 \\
\hline \multicolumn{3}{|l|}{$\begin{array}{l}\text { P00-P04 Feto e recém-nascido afetados por fatores maternos e por complicações da gravidez, } \\
\text { do trabalho de parto e do parto }\end{array}$} \\
\hline P01.0 Feto e recém-nascido afetados por incompetência do colo uterino & 193 & 4,9 \\
\hline \multicolumn{3}{|l|}{ Reduzíveis por adequado diagnóstico, tratamento, ações de promoção e imunização (A00-B99) (J00-J99) } \\
\hline \multicolumn{3}{|l|}{\begin{tabular}{|l|l|} 
A30-A49 Outras doenças bacterianas & \\
\end{tabular}} \\
\hline A41.9 Septicemia não especificada/choque séptico & 204 & 18,9 \\
\hline \multicolumn{3}{|l|}{ J09-J18 Influenza [gripe] e pneumonia } \\
\hline J18.9 Pneumonia não especificada & 204 & 18,9 \\
\hline \multicolumn{3}{|l|}{ A00-A09 Doenças infecciosas intestinais } \\
\hline A09 Diarreia e gastroenterite de origem infecciosa presumível & 121 & 11,2 \\
\hline \multicolumn{3}{|l|}{ Causas mal-definidas (R00-R99) (P00-P96) } \\
\hline \multicolumn{3}{|l|}{ R95-R99 Causas mal definidas e desconhecidas de mortalidade } \\
\hline R98 Morte sem assistência & 22 & 9,3 \\
\hline $\begin{array}{l}\text { R99 Outras causas mal definidas e as não especificadas de mortalidade/causas desconhecida } \\
\text { de morte }\end{array}$ & 114 & 48,1 \\
\hline \multicolumn{3}{|l|}{ P90-P96 Outros transtornos originados no período perinatal } \\
\hline P96.9 Afecções originadas no período perinatal não especificadas/debilidade congênita SOE & 67 & 28,3 \\
\hline \multicolumn{3}{|l|}{ Demais causas(Q00-Q99) } \\
\hline \multicolumn{3}{|l|}{ Q20-Q28 Malformações congênitas do aparelho circulatório } \\
\hline Q24.9 Malformação não especificada do coração & 336 & 13,7 \\
\hline \multicolumn{3}{|l|}{ Q00-Q07 Malformações congênitas do sistema nervoso } \\
\hline Q00.0 Anencefalia & 116 & 4,7 \\
\hline \multicolumn{3}{|l|}{ Q65-Q79 Malformações e deformidades congênitas do sistema osteomuscular } \\
\hline Q79.3 Gastrosquise & 104 & 4,2 \\
\hline
\end{tabular}

Tabela 3. Distribuição dos óbitos infantis segundo grupo de causas ${ }^{\mathrm{a}}$, grupo etário e peso ao nascer, Mato Grosso, 2007 a 2016.

\begin{tabular}{|c|c|c|c|c|c|c|c|c|c|}
\hline & \multirow[t]{2}{*}{$\mathbf{N}$} & \multicolumn{2}{|c|}{$\begin{array}{c}\text { Reduzíveis por } \\
\text { adequada atenção } \\
\text { à mulher na } \\
\text { gestação e parto e } \\
\text { ao recém-nascido }\end{array}$} & \multicolumn{2}{|c|}{$\begin{array}{c}\text { Reduzíveis } \\
\text { por adequado } \\
\text { diagnóstico, } \\
\text { tratamento, ações } \\
\text { de promoção e } \\
\text { imunização }\end{array}$} & \multicolumn{2}{|c|}{$\begin{array}{c}\text { Causas mal } \\
\text { definidas }\end{array}$} & \multicolumn{2}{|c|}{ Demais causas } \\
\hline & & $\mathbf{n}$ & $\%$ & $\mathbf{n}$ & $\%$ & $\mathbf{n}$ & $\%$ & $\mathbf{n}$ & $\%$ \\
\hline \multicolumn{10}{|l|}{ Grupo etário } \\
\hline Neonatal precoce & 3830 & 2922 & 76,3 & 15 & 0,4 & 56 & 1,5 & 837 & 21,9 \\
\hline Neonatal tardio & 1286 & 854 & 66,4 & 24 & 1,9 & 33 & 2,6 & 375 & 29,2 \\
\hline Pós-neonatal & 2616 & 177 & 6,8 & 1043 & 39,9 & 148 & 5,7 & 1248 & 47,7 \\
\hline \multicolumn{10}{|l|}{ Peso ao nascer $(\mathrm{g})^{\mathrm{b}}$} \\
\hline $500-1.499$ & 2473 & 1973 & 79,8 & 129 & 5,2 & 24 & 1 & 347 & 14 \\
\hline $1.500-2.499$ & 1655 & 851 & 51,4 & 151 & 9,1 & 39 & 2,4 & 614 & 37,1 \\
\hline$\geq 2.500$ & 2709 & 906 & 33,4 & 535 & 19,7 & 117 & 4,3 & 1151 & 42,5 \\
\hline
\end{tabular}

${ }^{a}$ Grupo de causas segundo a Lista de causa de mortes evitáveis por intervenções do Sistema Único de Saúde do Brasil (Malta et al. $\left.{ }^{15}\right)$; Informação faltante para causa básica do óbito para 9 registros $(0,12 \%)$; ${ }^{b}$ Informações faltantes para 895 registros $(11,6 \%)$. 
de mortalidade observados nas regiões Araguaia Xingu e Garças Araguaia.

Dentre as regiões de saúde que também apresentaram tendência decrescente da mortalidade infantil em Mato Grosso, chamou atenção a menor tendência de redução para a região de saúde da baixada cuiabana que possui a maior densidade demográfica, os maiores IDHs do estado e abriga os serviços de assistência à saúde de média e alta complexidade. Em contrapartida, a região Noroeste Matogrossense, caracterizada pela pequena extensão territorial, baixa densidade demográfica e piores indicadores socioeconômi$\cos ^{11}$, apresentou maior tendência de redução do coeficiente no estado.

O presente estudo alerta para a possível inversão da lógica de atendimento no SUS, em especial nas regiões com bons indicadores socioeconômicos, cuja porta de entrada deveria ser a atenção primária, porém os maiores investimentos ainda são direcionados para a média e alta complexidade. Espera-se que locais com melhores condições socioeconômicas e serviços de saúde ofereçam assistência de melhor qualidade e evitem óbitos precoces ${ }^{24}$. Em um município da Região Sul do Brasil, com elevado desenvolvimento econômico e social, foi encontrado efeito protetor dos indicadores socioeconômicos na determinação dos óbitos infantis ${ }^{25}$.

A prioridade em investir na "porta de entrada", não significa assegurar apenas o acompanhamento pré-natal de qualidade na atenção primária, havendo a necessidade de implementação do atendimento efetivo na rede de atenção a saúde materna infantil, como proposto pela Rede Cegonha ${ }^{26}$. Idealmente, os investimentos em saúde deveriam utilizar à lógica da equidade no financiamento em que se considerem indicadores importantes no direcionamento de recursos como o PIB per capita, o percentual de população com plano de saúde, com bolsa família, em extrema pobreza e a densidade demográfica dos municípios.

Outro aspecto encontrado no presente estudo e verificado em outros semelhantes ${ }^{27-29}$ que tem de ser considerado nos esforços para reduzir a mortalidade infantil no estado é o fato da maior parte dos óbitos infantis se concentrar no período neonatal, principalmente na primeira semana de vida. Em 1990, a taxa de mortalidade neonatal no Brasil era de 23,1 óbitos por $1.000 \mathrm{NV}$ e a taxa de mortalidade pós-neonatal era de 24/1.000 NV; em 2015, as mesmas taxas foram de 9,9/1.000 NV e 4,3/1.000 NV, respectivamente ${ }^{8}$.

As doenças infectocontagiosas prevalentes no período pós-neonatal reduziram devido melho- rias nas condições socioeconômicas e ambientais da população, por conseguinte, aumentaram as causas de morte no período neonatal relacionadas, em especial, às condições de assistência à saúde da mulher, prestada antes e durante a gestação, e ao recém-nascido, no pré/pós-parto ${ }^{24}$, como foi verificado no estado de Mato Grosso.

A redução na taxa de mortalidade infantil, em especial da pós-neonatal, evitáveis por ações adequadas de diagnóstico, tratamento, ações de promoção e imunização, indica o quanto as medidas sanitárias contribuíram para a reorganização do modelo de atenção a saúde no Brasil e impactaram positivamente a situação da saúde no país, principalmente na população infantil ${ }^{21}$. Nesse aspecto, destaca-se a implantação de programas de controle de doenças prevalentes em crianças e a introdução de novas vacinas, além de outros determinantes como a atuação da Estratégia de Saúde da Família ${ }^{30,31}$.

Diante do elevado coeficiente de mortalidade neonatal e da emergência de ações que visam a redução das causas evitáveis, o estado de Mato Grosso, juntamente com outros nove estados da federação, passaram a compor a estratégia qualiNEO. A estratégia integra diversas políticas de redução da mortalidade infantil e visa qualificar as práticas de atenção ao recém-nascido voltada para a redução das taxas de mortalidade neonatal e de asfixia ao nascimento ${ }^{32}$.

$\mathrm{O}$ atual cenário alerta para a necessidade de melhorar o acesso aos serviços de saúde e a qualidade da atenção à mulher e ao recém-nascido, em especial aos cuidados intensivos neonatais após o parto. Estudos indicam que evitar partos prematuros decorrentes de cesarianas desnecessárias e aperfeiçoar o manejo obstétrico das complicações do parto, bem como da assistência neonatal, podem contribuir para a redução das TMI por causas evitáveis reduzíveis por adequada atenção à mulher na gestação, parto e ao recém-nascido ${ }^{33,34}$.

No presente estudo, aproximadamente $80 \%$ dos óbitos entre os nascidos com 500 a 1.499 gramas foram classificados no grupo de causas "reduzíveis por adequada atenção à mulher na gestação e parto e ao recém-nascido". A maior concentração e o aumento da proporção de óbitos evitáveis de recém-nascidos com baixo peso ao nascer nesse grupo de causa indica a necessidade de rever a importância dessa variável dentre os critérios de evitabilidade, pois, sabe-se que o peso ao nascer é um fator isolado determinante para a sobrevivência infantil ${ }^{30,33}$.

A associação entre baixo peso e prematuridade já foi evidenciada na literatura e ambas as 
condições são, em parte, preveníveis por cuidados pré-natais de qualidade ${ }^{35,36}$. Estudo de base populacional em Minas Gerais indicou que apenas $26,8 \%$ dos acompanhamentos pré-natais foram classificados como adequado ${ }^{37}$. Esses resultados sugerem que não basta apenas ampliar o acesso aos níveis de atenção à saúde, é necessário investir na melhoria da qualidade da assistência pré-natal.

Destaca-se no estudo, a concentração e a tendência crescente da proporção de óbitos por causas não claramente evitáveis $(31,8 \%)$, neste grupo, destacou-se os óbitos por malformações congênitas do aparelho circulatório $(13,7 \%)$, do sistema nervoso - anencefalia $(4,7 \%)$ e do sistema osteomuscular - gastrosquise $(4,2 \%)$. $\mathrm{O}$ achado corrobora ao observado em Mato Grosso do Sul, com predominância de $24,3 \%$ de óbitos pelo grupo de causas não claramente evitáveis, sendo $14,9 \%$ do total de óbitos causados por malformações congênitas ${ }^{38}$. No Paraná também foi observado maior frequência de malformações congênitas não especificadas nesse grupo de causa, seguido das malformações do aparelho circulatório $^{39}$. Os resultados também são similares ao estudo realizado em Cuiabá-MT em 2007, no qual foi verificado maior ocorrência de anencefalia $(17,7 \%)$ entre as causas não claramente evitáveis ${ }^{28}$.

Neste contexto, a vigilância dos óbitos infantis é reconhecida como importante estratégia para avaliar as políticas de redução da mortalidade precoce e evitável, e também para qualificar a causa básica do óbito, em especial daqueles codificados como causas mal definidas e demais causas.

O Ministério da saúde incentiva à atuação dos Comitês de investigação do óbito nas três esferas de governo federal, estadual e municipal e nos serviços de saúde públicos e privados, para compreender as circunstâncias de ocorrência dos óbitos, identificar fatores de risco e definir políticas públicas, em particular, dirigidas a redução da mortalidade materna e infantil ${ }^{40}$.

Na capital do estado de Mato Grosso o Comitê Municipal de Mortalidade Materna e Infantil e a equipe da Vigilância de Nascimentos e Óbitos foram reestruturados em 2006 com o objetivo de qualificar os dados relativos aos óbitos maternos e infantis e planejar ações pertinentes. A partir da referida reestruturação, a proporção de óbitos totais investigados pela Vigilância de Óbito do município passou de $15 \%$ em 2006, para $81 \%$ em $2007^{28}$. Mais recentemente, em 2015, estimou-se a proporção $96 \%$ de óbitos investigados ${ }^{41}$.

Apesar dos avanços para institucionalização da vigilância dos óbitos infantis no estado, aprimorado com a identificação de sua causa segundo critério de evitabilidade, há muito que ser aprimorado e instituído para que se possa dispor de informações de qualidade referente às ações e serviços de saúde disponibilizados para esta parcela da população (mulheres, gestantes e crianças em seu primeiro ano de vida).

Ressalta-se que este estudo, embora apresente vantagens por utilizar dados secundários, em relação a sua disponibilidade, encontra limitações relacionadas a possíveis incompletudes e inconsistência na qualificação das causas básicas do óbito. Devido à importância do indicador de mortalidade infantil, há necessidade de novos estudos para identificar quais os fatores associados aos óbitos evitáveis por intervenção do Sistema Único de Saúde.

O conhecimento das causas de morte infantil no estado, especialmente daquelas reduzíveis por ações efetivas dos serviços de saúde, são iniciativas imprescindíveis para o planejamento de ações que visem diminuir as desigualdades regionais $\mathrm{e}$ melhorar a vigilância do óbito. Mais do que a implantação de tecnologias sofisticadas, a melhoria dos indicadores de mortalidade infantil pode ser alcançada com o aperfeiçoamento de rotinas de atendimento na atenção básica.

Uma dificuldade adicional é definir o tamanho da responsabilidade do setor saúde e das ações intersetoriais na redução desse indicador frente à redução do orçamento da saúde, da educação e das políticas assistenciais. Uma vez que para manter a tendência favorável de redução de mortes precoces faz-se necessário que as políticas e serviços de saúde com foco na qualidade da atenção e na melhoria das condições de saúde sejam mantidos ou reforçados. 


\section{Colaboradores}

Todos os autores asseguram a participação na concepção do estudo para tornar pública a nossa responsabilidade pelo seu conteúdo.

\section{Financiamento}

O presente trabalho foi realizado com apoio da Coordenação de Aperfeiçoamento de Pessoal de Nível Superior - Brasil (CAPES).

\section{Referências}

1. Organização Pan-Americana da Saúde (OPAS). Indicadores de Salud: elementos básicos para el análisis de la situación de salud. Boletín Epidemiol 2001; 22(4):15.

2. Rede Interagencial de Informação para a saúde (RIPSA). Indicadores Básicos para a saúde no Brasil: conceitos e aplicações. 2a ed. Brasília: OPAS; 2008.

3. Brasil. Ministério da Saúde (MS). Pacto pela redução da mortalidade infantil no Nordeste e Amazônia Legal: 2009-2010 [documento na Internet]. Brasília: MS; 2010. [acessado 2017 Ago 25]. Disponível em: http:// bvsms.saude.gov.br/bvs/publicacoes/pacto_reducao_ mortalidade_infantil_nordeste.pdf

4. Rutstein DD, Berenberg W, Chalmers TC, Child CG, Fishman AP, Perrin EB. Measuring the quality of medical care: a clinical method. $N$ Engl J Med 1976; 294(11):582-588.

5. Suárez-Varela MM, Llopis GA, Tejerizo PML. Variations in avoidable mortality in relation to health care resources and urbanization level. J Environ Pathol Toxicol Oncol 1996; 15(2-4):149-154.

6. Maranhão AGK, Vasconcelos AMN, Porto DL, França E. Mortalidade infantil no Brasil: tendências, componentes e causas de morte no período de 2000 a 2010 In: Brasil. Ministério da Saúde (MS). Secretaria de Vigilância em Saúde. Departamento de Análise de Situação de Saúde. Saúde Brasil 2011: uma análise da situação de saúde e a vigilância da saúde da mulher. Brasília: Editora MS; 2011.p. 163-182.

7. World Health Organization (WHO). Newborns: reducing mortality [página na Internet]. [acessado 2017 Jan 10]. Disponível em: http://www.who.int/mediacentre/factsheets/fs333/en/

8. Brasil. Ministério da Saúde (MS). Secretaria de Vigilância em Saúde. Departamento de Vigilância de Doenças e Agravos Não Transmissíveis e Promoção da Saúde. Saúde Brasil 2015/2016: uma análise da situação de saúde e da epidemia pelo vírus Zika e por outras doenças transmitidas pelo Aedes aegypti. Brasília: MS; 2017.
9. Brasil. Portal Brasil (ONU): Brasil cumpre meta de redução da mortalidade infantil [página na Internet]. 2015 [acessado 2017 Out 15]. Disponível em: http:// www.casacivil.gov.br/central-de-conteudos/noticias/2015/setembro/onubrasil-cumpre-meta-de-reducao-da-mortalidade-infantil

10. Associação Brasileira de Saúde Coletiva (ABRASCO). Especial Abrasco sobre o aumento da mortalidade infantil e materna no Brasil [página na Internet]. 2018 [acessado 2018 Ago 31]. Disponível em: https://www. abrasco.org.br/site/outras-noticias/institucional/especial-abrasco-sobre-o-aumento-da-mortalidade-infantil-e-materna-no-brasil/36777/

11. Scatena JHG, Kehrig RT, Spinelli MA. Regiões de Saúde: diversidade e processo de regionalização em Mato Grosso. São Paulo: Hucitec; 2014.

12. Instituto Brasileiro de Geografia e Estatística (IBGE). Estatística da população por cidades e estados [página na Internet]. [acessado 2017 Ago 22]. Disponível em: https://www.ibge.gov.br/estatisticas-novoportal/porcidade-estado-estatisticas.html? $\mathrm{t}=$ destaques $\& \mathrm{c}=51$

13. Instituto Brasileiro de Geografia e Estatística (IBGE). Censo Demográfico 2010 - Mato Grosso [página na Internet]. [acessado 2017 Ago 22]. Diponível emhttps://censo2010.ibge.gov.br/sinopse/index.php?dados $=29 \& u f=51$

14. Brasil. Ministério da Saúde (MS). Secretaria de Vigilância em Saúde. Sistema nacional de vigilância em saúde: relatório de situação: Mato Grosso. $5^{\mathrm{a}}$ ed. Brasília: MS; 2011.

15. Malta DC, Sardinha LMV, Moura L, Lansky S, Leal MC, Szwarcwald CL, França E, Almeida MF, Duarte EC. Atualização da lista de causas de mortes evitáveis por intervenções do Sistema Único de Saúde do Brasil. Epidemiol Serv Saúde 2010; 19(2):173-176.

16. Stevanato JM, Gaíva MAM, Silva AMC. Tendência da cobertura do Sistema de Informações sobre Nascidos Vivos em Mato Grosso, 2000 a 2012. Epidemiol Serv Saúde 2017; 26(2):265-274. 
17. Oliveira CM, Bonfim CV, Guimarães MJB, Frias PG, Medeiros ZM. Mortalidade infantil: tendência temporal e contribuição da vigilância do óbito. Acta Paul Enferm 2016; 29(3):282-290.

18. Areco KCN, Konstantyner T, Taddei JAAC. Secular trends in infant mortality by age-group and avoidable components in the State of São Paulo, 1996-2012. Rev Paul Pediatr 2016; 34(3):263-270.

19. Bando DH, Kawano MK, Kumagai LT, Gouveia JLV, Reis TM, Bernardo ES, Patronieri AT. Tendência das taxas de mortalidade infantil e de seus componentes em Guarulhos-SP, no período de 1996 a 2011. Epidemiol Serv Saúde 2014; 23(4):767-772.

20. Frias PG, Szwarcwald CL, Morais Neto OL, Leal MC, Cortez-Escalante JJ, Souza Junior PRB, Almeida WS, Silva Junior JB. Utilização das informações vitais para a estimação de indicadores de mortalidade no Brasil: da busca ativa de eventos ao desenvolvimento de métodos. Cad Saúde Pública 2017; 33(3):e00206015.

21. United Nations Children's Fund (UNICEF). World Health Organization (WHO). World Bank Group. United Nations (UN). Levels \& Trends in Child Mortality: Report 2015. Estimates Developed by the UN Inter-agency Group for Child Mortality Estimation [documento na Internet]. 2015 [acessado 2018 Ago 25]. Disponível em: https://www.unicef.org/media/files/ IGME_report_2015_child_mortality_final.pdf

22. Souza LG, Santos RV, Coimbra JR. Estrutura etária, natalidade e mortalidade do povo indígena Xavante de Mato Grosso, Amazônia, Brasil. Cien Saude Colet 2010; 15(1):1465-1473.

23. Instituto Brasileiro de Geografia e Estatística (IBGE). Censo Demográfico de 2010. Características Gerais dos Indígenas. Resultados do Universo. Rio de Janeiro: IBGE; 2012.

24. Victora CG, Aquino EM, Carmo Leal M, Monteiro CA, Barros FC, Szwarcwald CL. Maternal and child health in Brazil: progress and challenges. Lancet 2011; 377(9780):1863-1876.

25. Kropiwiec MV, Selma CF, Augusto RA. Factors associated with infant mortality in a Brazilian city with high human development index. Rev Paul Pediatr 2017; 35(4):391-398.

26. Brasil. Portaria $\mathrm{n}^{\mathrm{o}} 1.459$, de 24 de junho de 2011. Institui no âmbito do Sistema Único de Saúde - SUS - a Rede Cegonha. Diário Oficial da União; 2011.

27. Nascimento SG, Oliveira CM, Sposito V, Ferreira DKS, Bonfim CV. Mortalidade infantil por causas evitáveis em uma cidade do Nordeste do Brasil. Rev Bras Enferm 2014; 67(2):208-212.

28. Lourenço EC, Brunken GS, Luppi CG. Mortalidade infantil neonatal: estudo das causas evitáveis em Cuiabá, Mato Grosso, 2007. Epidemiol Serv Saúde 2013; 22(4):697-706.

29. Corassa RB, Alves PAB, Rodrigues AC, Machado GVC. Tendência da Mortalidade Neonatal na Macrorregião do Jequitinhonha, 2000 a 2011. Rev Bras Pesq 2014; 16(3):73-83.

30. Malta DC, Duarte EC, Escalante JJC, Almeida MF, Sardinha LMV, Macário EM, Monteiro RA, Morais Neto OL. Mortes evitáveis em menores de um ano, Brasil, 1997 a 2006: contribuições para a avaliação de desempenho do Sistema Único de Saúde. Cad Saúde Pública 2010; 26(3):481-491.
31. Ferrari RAP, Bertolozzi MR. Mortalidade pós-neonatal no território brasileiro: uma revisão da literatura. Rev Esc Enferm USP 2012; 46(5):1207-1214.

32. Brasil. Ministério da Saúde (MS). Estratégia QualiNEO [página na Internet]. [acessado 2017 Out 12]. Disponível em: http://portalsaude.saude.gov.br/index.php/o-ministerio/principal/secretarias/sas/sasnoticias/29023-ministerio-da-saude-lanca-estrategia -para-reduzir-mortalidade-neonatal

33. Santos IS, Matijasevich A, Gorgot LRMR, Valle NCJ, Menezes AM. Óbitos infantis evitáveis nas coortes de nascimentos de Pelotas, Rio Grande do Sul, Brasil, de 1993 e 2004. Cad Saúde Pública 2014; 30(11):23312343.

34. França EB, Lansky S, Rego MAS, Malta DC, França JS, Teixeira R, Porto D, Almeida MF, Souza MFM, Szwarcwald CL, Mooney M, Naghavi M, Vasconcelos AMN. Principais causas da mortalidade na infância no Brasil, em 1990 e 2015: estimativas do estudo de Carga Global de Doença. Rev Bras Epidemiol 2017; 20(Supl. 1):46-60.

35. Frederick IO, Williams MA, Sales AE, Martin DP, Killien M. Pre-pregnancy body mass index, gestational weight gain, and other maternal characteristics in relation to infant birth weight. Matern Child Health J 2008; 12(5):557-567.

36. Silveira MF, Santos IS, Barros AJD, Matijasevich A, Barros FC, Victora CG. Aumento da prematuridade no Brasil: revisão de estudos de base populacional. Rev Saude Publica 2008; 42(5):957-964.

37. Lisboa L, Abreu DMX de, Lana AMQ, França EB. Mortalidade infantil: principais causas evitáveis na região Centro de Minas Gerais, 1999-2011. Epidemiol Serv Saúde 2015; 24(4):711-720.

38. Gastaud ALGS, Honer MR, Cunha RV. Mortalidade infantil e evitabilidade em Mato Grosso do Sul, Brasil, 2000 a 2002. Cad Saúde Pública 2008; 24(7):16311640.

39. Dutra LS, Ferreira AP. Associação entre malformações congênitas e a utilização de agrotóxicos em monoculturas no Paraná, Brasil. Saúde Debate 2017; 41(esp. 2):241-253.

40. Brasil. Ministério da Saúde (MS). Portaria no 1.258 , de 28 de junho de 2004. Institui o Comitê Nacional de Prevenção do Óbito Infantil e Fetal. Diário Oficial da União; 2004.

41. Brasil. Ministério da Saúde (MS). Sistema de Informação sobre Mortalidade - DATASUS [página na Internet]. [acessado 2017 Jan 12]. Disponível em: http://tabnet.datasus.gov.br/cgi/tabcgi.exe?sim/cnv/inf10mt. def

Artigo apresentado em 03/07/2018

Aprovado em 20/11/2018

Versão final apresentada em 22/11/2018 